\title{
Parallel Implementation of Large-Scale Linear Scaling Density Functional Theory Calculations With Numerical Atomic Orbitals in HONPAS
}

\author{
Zhaolong Luo, Xinming Qin *, Lingyun Wan, Wei Hu* and Jinlong Yang
}

Hefei National Laboratory for Physical Sciences at the Microscale, Department of Chemical Physics, and Synergetic Innovation Center of Quantum Information and Quantum Physics, University of Science and Technology of China, Hefei, China

OPEN ACCESS

Edited by:

Hans Martin Senn,

University of Glasgow,

United Kingdom

Reviewed by:

Ma Yingjin,

Computer Network Information Center

(CAS), China

C. Y. Yam,

Beijing Computational Science

Research Center, China

*Correspondence:

Xinming Qin

xmain03@ustc.edu.cn

Wei Hu

whuustc@ustc.edu.cn

Specialty section:

This article was submitted to Theoretical and Computational

Chemistry

a section of the journal

Frontiers in Chemistry

Received: 31 July 2020 Accepted: 08 September 2020 Published: 26 November 2020

Citation:

Luo Z, Qin X, Wan L, Hu W and Yang J (2020) Parallel Implementation of Large-Scale Linear Scaling Density

Functional Theory Calculations With

Numerical Atomic Orbitals in HONPAS. Front. Chem. 8:589910. doi: 10.3389/fchem.2020.589910
Linear-scaling density functional theory (DFT) is an efficient method to describe the electronic structures of molecules, semiconductors, and insulators to avoid the high cubic-scaling cost in conventional DFT calculations. Here, we present a parallel implementation of linear-scaling density matrix trace correcting (TC) purification algorithm to solve the Kohn-Sham (KS) equations with the numerical atomic orbitals in the HONPAS package. Such a linear-scaling density matrix purification algorithm is based on the Kohn's nearsightedness principle, resulting in a sparse Hamiltonian matrix with localized basis sets in the DFT calculations. Therefore, sparse matrix multiplication is the most time-consuming step in the density matrix purification algorithm for linear-scaling DFT calculations. We propose to use the MPI_Allgather function for parallel programming to deal with the sparse matrix multiplication within the compressed sparse row (CSR) format, which can scale up to hundreds of processing cores on modern heterogeneous supercomputers. We demonstrate the computational accuracy and efficiency of this parallel density matrix purification algorithm by performing large-scale DFT calculations on boron nitrogen nanotubes containing tens of thousands of atoms.

Keywords: linear-scaling density functional theory, density matrix purification algorithm, sparse matrix multiplication, parallel implementation, tens of thousands of atoms

\section{INTRODUCTION}

The Kohn-Sham density functional theory (DFT) (Hohenberg and Kohn, 1964; Kohn and Sham, 1965) has been successfully applied to perform first-principles calculations for describing the electronic structures of both molecules and solids. However, conventional DFT calculations based on direct diagonalization methods for solving the KS equations have a high cubic-scaling cost (Goedecker, 1999), which can usually be used to study medium-scale systems containing up to hundreds of atoms. Therefore, it is difficult to achieve massive parallelism for these conventional cubic-scaling methods due to complex communication issues. To avoid the bottleneck arising from the computational cost and memory usage of directly diagonalizing the Hamiltonian matrix in conventional DFT calculations, linear-scaling methods using local basis functions 
have been proposed (Goedecker, 1999), strongly promoting the applications of DFT calculations in large-scale systems containing thousands of atoms.

In general, linear-scaling methods include direct, variational, and purification methods (Bowler and Miyazaki, 2012). The direct methods are featured by direct evaluation of density matrix using various approximations, including divide and conquer (Yang, 1991; Yang and Lee, 1995) and Fermi operator expansion (Goedecker and Colombo, 1994; Goedecker and Teter, 1995; Liang et al., 2003). The variational methods minimize the total energy with respect to the auxiliary density matrix or Wannier-like orbitals, covering density matrix minimization method (Daw, 1993; Li et al., 1993; Nunes and Vanderbilt, 1994) and orbital minimization method (OMM) (Galli and Parrinello, 1992; Mauri and Galli, 1994; Kim et al., 1995; Ordejón et al., 1995). The third scheme exploits the purification polynomial and iterative solution, which is known as density matrix purification method (Palser and Manolopoulos, 1998; Niklasson, 2002; Niklasson et al., 2003). Nearly all of the linear-scaling methods are based on the Kohn's nearsightedness principle with localized basis sets, such as Gaussian type orbitals (GTOs) (Frisch et al., 1984) and numerical atomic orbitals (Shang et al., 2010) (NAOs), resulting in the sparsity of density matrix with a number of non-zero entries that increase linearly with the system size, so the linear-scaling matrix-matrix multiplication can be achieved (VandeVondele et al., 2012; Kim and Jung, 2016). In particular, the density matrix purification algorithms without prior knowledge of the chemical potential, including the trace-preserving canonical purification scheme of Palser and Manolopoulos (PM) (Palser and Manolopoulos, 1998; Daniels and Scuseria, 1999), the trace-correcting purification (TC) (Niklasson, 2002), and the trace resetting density matrix purification (TRS) (Niklasson et al., 2003), have been demonstrated as accurate and efficient linear-scaling methods to describe the electronic structures of molecules, semiconductors, and insulators. However, almost all of the developed linear-scaling techniques (direct, variational, and purification methods) assume the presence of a non-zero gap in the electronic structure, which prevents them from treating metallic systems. Recently, Suryanarayana (2017) have employed the $O(N)$ Spectral Quadrature (SQ) method (Suryanarayana, 2013; Pratapa et al., 2016) to study the locality of electronic interactions in aluminum (a prototypical metallic system) as a function of smearing/electronic temperature. They have found exponential convergence accompanied by a rate that increases sub-linearly with smearing. It is also worth mentioning that all these linearscaling methods based on Kohn's nearsightedness principle are limited to the localization of density matrix (Bowler and Miyazaki, 2012). A recently published innovative version of PEXSI scheme named iPEXSI (Etter, 2020), which does not rely on the nearsightedness principle, can scale provably better than cubically even in the absence of localization of density matrix. The iPEXSI algorithm utilizes a localization property of triangular factorization, which could extend the usable range of linear-scaling method to metallic system without the constraint of finite electronic temperature.
Nowadays, with the rapid development of modern heterogeneous supercomputers, the high-performance computing (HPC) has become a powerful tool for accelerating the DFT calculations to deal with large-scale systems. Several highly efficient DFT software based on low-scaling methods have been developed, such as SIESTA (Soler et al., 2002), OPENMX (Ozaki and Kino, 2005), CP2K (Kühne et al., 2020), CONQUEST (Gillan et al., 2007), PROFESS (Ho et al., 2008), FREEON (Challacombe, 2014), ONETEP (Skylaris et al., 2005), BigDFT (Genovese et al., 2008; Mohr et al., 2014), FHI-aims (Blum et al., 2009), ABACUS (Chen et al., 2010, 2011), HONPAS (Qin et al., 2015), and DGDFT (Lin et al., 2012; Hu et al., 2015a,b; Banerjee et al., 2016; Zhang et al., 2017), which are capable to make full advantage of the massive parallelism available on HPC architectures beneting from the local data communication of sparse Hamiltonian matrix generated with local basis sets. In linear-scaling DFT calculations, the kernel for HPC is to parallel sparse matrix-matrix multiplication. In order to realize the HPC parallelism, two massively parallel libraries of BCSR (Borštnik et al., 2014) and NTPOLY (Dawson and Nakajima, 2018) have been developed, which have shown a high performance for the density matrix purification algorithms implemented in the CP2K (Kühne et al., 2020) and CONQUEST (Gillan et al., 2007) packages.

In this work, we present a parallel implementation of linearscaling density matrix second-order trace-correcting purification (TC2) algorithm (Niklasson, 2002) to solve the KS equations with the NAOs in the HONPAS package (Qin et al., 2015). We propose to use the MPI_Allgather function for parallel programming to deal with such sparse matrix multiplication within the CSR format, which can be scaled linearly up to hundreds of processing cores on modern heterogeneous supercomputers. We demonstrate the computational accuracy and efficiency of this linear-scaling density matrix purification method by performing large-scale DFT calculations on boron nitrogen nanotubes containing thousands of atoms.

\section{METHODOLOGY}

\subsection{Density Functional Theory}

We first give a brief review of Kohn-Sham density functional theory (KS-DFT). The key spirit of KS-DFT is to solve the KS equations defined as

$$
\hat{H} \psi_{i}(\mathbf{r})=\left(\hat{T}+\hat{V}_{\text {ion }}+\hat{V}_{\mathrm{H}}+\hat{V}_{\mathrm{xc}}\right) \psi_{i}(\mathbf{r})=\varepsilon_{i} \psi_{i}(\mathbf{r})
$$

where $\hat{H}$ is the Hamiltonian operator, $\psi_{i}$ is the $i$ th molecular orbital, and $\epsilon_{i}$ is the corresponding orbital energy. $\hat{T}$ is the kinetic operator, $\hat{V}_{\text {ion }}$ is the ionic potential operator, and $\hat{V}_{\mathrm{H}}$ is the Hartree potential operator defined as

$$
\hat{V}_{\mathrm{H}}(\mathbf{r})=\int \frac{\rho\left(\mathbf{r}^{\prime}\right)}{\left|\mathbf{r}-\mathbf{r}^{\prime}\right|} d \mathbf{r}^{\prime}
$$

where the electron density is given by

$$
\rho(\mathbf{r})=\sum_{i=1}^{N_{e}} \psi_{i}^{*}(\mathbf{r}) \psi_{i}(\mathbf{r})
$$


In the approximation of linear combination of atomic orbitals (LCAO) (Mulliken, 1955), the $\psi_{i}$ is expanded on a set of NAOs $\left\{\phi_{\mu}(r)\right\}_{\mu=1}^{N_{\mathrm{b}}}$

$$
\psi_{i}(\mathbf{r})=\sum_{\mu}^{N_{\mathrm{b}}} c_{\mu i} \phi_{\mu}(\mathbf{r})
$$

where $c_{\mu i}$ is the expansion coefficient at the $\mu$ th atomic orbital and $N_{\mathrm{b}}$ is the number of NAOs. Then, the KS equations can be rewritten into matrix notations as

$$
H C=S C E
$$

where $C$ is coefficient matrix and $E$ is the corresponding orbital energy. $H$ and $S$ are the Hamiltonian and overlap matrices over the NAOs

$$
\begin{aligned}
H_{\mu \nu} & =\int \phi_{\mu}^{*}(\mathbf{r}) \hat{H} \phi_{\nu}(\mathbf{r}) d \mathbf{r} \\
S_{\mu \nu} & =\int \phi_{\mu}^{*}(\mathbf{r}) \phi_{\nu}(\mathbf{r}) d \mathbf{r}
\end{aligned}
$$

The default choice in the SIESTA package is to use the direct diagonalization method though the LAPACK and ScaLAPACK libraries to solve this eigenvalue problem with a high cubicscaling cost. Therefore, the computational cost and memory usage of such DFT calculations increase rapidly as the system size, which are only limited to small systems containing hundreds of atoms. In order to overcome this limitation, several linearscaling methods have been implemented in the SIESTA package, such as the Kim-Mauri-Galli (KMG) orbital minimization (OMM) method (Galli and Parrinello, 1992; Mauri and Galli, 1994; Kim et al., 1995; Corsetti, 2014) and divide and conquer method (Cankurtaran et al., 2008). The KMG requires a initial approximate Wannier functions and a prior knowledge of the chemical potential. In the HONPAS-SIESTA package (Qin et al., 2015), we implement the density matrix purification algorithms, including the trace-preserving canonical purification scheme of PM (Palser and Manolopoulos, 1998; Daniels and Scuseria, 1999), the trace-correcting purification (TC) (Niklasson, 2002), and the trace resetting density matrix purification (TRS) (Niklasson et al., 2003).

\subsection{Linear-Scaling Density Matrix Purification Algorithms}

After constructing the Hamiltonian matrix, the density matrix can be obtained by directly diagonalizing the Hamiltonian matrix with cubic-scaling cost. In order to avoid the high cost of explicit diagonalization, we implement three density matrix purification algorithms, without prior knowledge of the chemical potential for linear-scaling DFT calculations, including the trace-preserving canonical purification scheme of PM, the trace-correcting purification (TC) (Niklasson, 2002), and the trace resetting density matrix purification (TRS) (Niklasson et al., 2003), in the HONPAS package (Qin et al., 2015). In this work, we use the second-order trace-correcting purification (TC2) (Niklasson, 2002) algorithm with orthogonal basis sets to illustrate our parallel algorithms. In the coordinate presentation, the general form of density matrix can be given by

$$
\rho\left(\mathbf{r}, \mathbf{r}^{\prime}\right)=\sum_{i=1}^{N_{b}} f\left(\varepsilon_{i}\right) \psi_{i}(\mathbf{r}) \psi_{i}^{*}\left(\mathbf{r}^{\prime}\right)
$$

where $f\left(\varepsilon_{i}\right)$ is the Fermi distribution function of energy level $\varepsilon_{i}$ at finite electronic temperature

$$
f\left(\varepsilon_{i}\right)=\frac{1}{1+e^{\beta\left(\varepsilon_{i}-\mu\right)}}
$$

with the chemical potential $\mu$ and the inverse temperature $\beta=$ $1 / k_{B} T$. Within the LCAO method, we can transform the density matrix from coordinate presentation to the basis presentation, then the density matrix element $P_{\mu \nu}$ becomes:

$$
\begin{aligned}
P_{\mu \nu} & =\int \phi_{\mu}^{*}(\mathbf{r}) \rho\left(\mathbf{r}, \mathbf{r}^{\prime}\right) \phi_{\nu}\left(\mathbf{r}^{\prime}\right) d \mathbf{r} d \mathbf{r}^{\prime} \\
& =\int \phi_{\mu}^{*}(\mathbf{r}) \sum_{i=1}^{N_{\mathrm{b}}} f\left(\varepsilon_{i}\right) \psi_{i}(\mathbf{r}) \psi_{i}^{*}\left(\mathbf{r}^{\prime}\right) \phi_{\nu}\left(\mathbf{r}^{\prime}\right) d \mathbf{r} d \mathbf{r}^{\prime} \\
& =\sum_{i=1}^{N_{b}} f\left(\varepsilon_{i}\right) \sum_{\lambda}^{N_{\mathrm{b}}} c_{\lambda i} \sum_{\kappa}^{N_{\mathrm{b}}} c_{\kappa i}^{*} \int \phi_{\mu}^{*}(\mathbf{r}) \phi_{\lambda}(\mathbf{r}) d \mathbf{r} \int \phi_{\kappa}^{*}\left(\mathbf{r}^{\prime}\right) \phi_{\nu}\left(\mathbf{r}^{\prime}\right) d \mathbf{r}^{\prime} \\
& =\sum_{i=1}^{N_{b}} f\left(\varepsilon_{i}\right) \sum_{\lambda}^{N_{\mathrm{b}}} c_{\lambda i} \sum_{\kappa}^{N_{\mathrm{b}}} c_{\kappa i}^{*} S_{\mu \lambda} S_{\nu \kappa}
\end{aligned}
$$

If NAOs are orthogonal, the density matrix element $P_{\mu \nu}$ can be written as

$$
P_{\mu \nu}=\sum_{i}^{N_{b}} f\left(\varepsilon_{i}\right) c_{\mu i} c_{v i}^{*}
$$

Note that $\varepsilon_{i}$ is relative to the eigenvalue of $\hat{H} \psi_{i}=\varepsilon_{i} \psi_{i}$, so $P_{\mu \nu}$ can be rewritten as

$$
\begin{aligned}
P_{\mu \nu} & =\int \phi_{\mu}^{*}(\mathbf{r}) \rho\left(\mathbf{r}, \mathbf{r}^{\prime}\right) \phi_{\nu}\left(\mathbf{r}^{\prime}\right) d \mathbf{r} d \mathbf{r}^{\prime} \\
& =\sum_{i}^{N_{b}} \int \phi_{\mu}^{*}(\mathbf{r}) f\left(\varepsilon_{i}\right) \psi_{i}(\mathbf{r}) \psi_{i}^{*}\left(\mathbf{r}^{\prime}\right) \phi_{\nu}\left(\mathbf{r}^{\prime}\right) d \mathbf{r} d \mathbf{r}^{\prime} \\
& =\int \phi_{\mu}^{*}(\mathbf{r}) f(\hat{H}) \sum_{i}^{N_{b}}\left(\psi_{i}(\mathbf{r}) \psi_{i}^{*}\left(\mathbf{r}^{\prime}\right)\right) \phi_{\nu}\left(\mathbf{r}^{\prime}\right) d \mathbf{r} d \mathbf{r}^{\prime} \\
& =\int \phi_{\mu}^{*}(\mathbf{r}) f(\hat{H}) \phi_{\nu}\left(\mathbf{r}^{\prime}\right) d \mathbf{r} d \mathbf{r}^{\prime}
\end{aligned}
$$

which implies that $P$ is commutative with $H$, namely $[H, P]=0$. Another substantial property of the appropriate density matrix is particle conservation, $\operatorname{Tr}(P)=N_{e} / 2$.

When the electronic temperature is zero, $f\left(\varepsilon_{i}\right)=1$ and the density matrix of insulator can be written as

$$
P_{\mu \nu}=\sum_{i}^{N_{e}} c_{\mu i} c_{\nu i}^{*}
$$




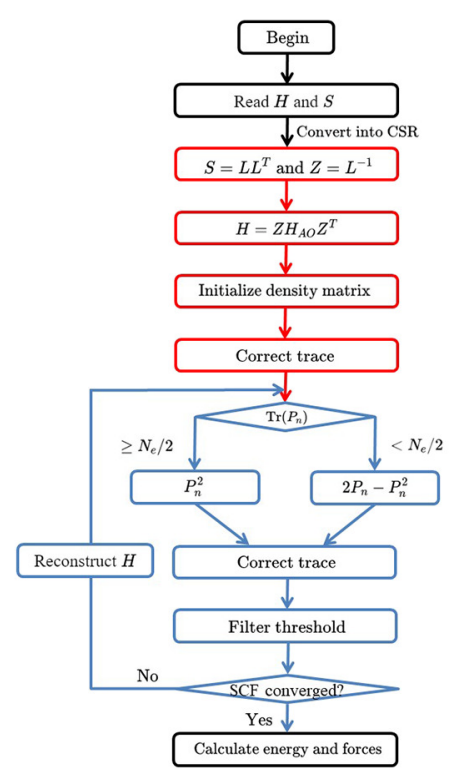

FIGURE 1 | The flowchart of density matrix purification TC2 method. There are four time-consuming parts in the TC2 method, including constructing the Hamiltonian matrix, initializing the density matrix $P$ from the Hamiltonian matrix with Cholesky and Lanzos methods, updating the density matrix with parallel sparse matrix-matrix multiplication, and computing total energy and atomic forces after SCF iterations.

which must satisfy the so-called idempotency $P P=P$ with an orthogonal basis.

The solution of eigenvalue problem is under three restricted conditions of the density matrix mentioned above, which is known as the purification method. The trace-preserving canonical purification scheme of PM (Palser and Manolopoulos, 1998), which imposes commutation relation and tracepreserving condition, works with a predefined occupation and does not need the input or adjustment of the chemical potential. Trace-conserving spectral projections are performed during each iteration, until the density matrix $P_{n}$ converges to the correct one that satisfies the idempotency condition. This method is inefficient at low and high partial occupancies (Palser and Manolopoulos, 1998; Daniels and Scuseria, 1999). A subsequent strategy proposed by Niklasson named TC algorithm (Niklasson, 2002). Its second-order form is called the second-order tracecorrecting purification (TC2) method (Niklasson et al., 2003). The higher order TC2 requires additional matrix multiplications, which pursues a more rapid reduction of errors and a less step of purification iterations (Kim and Jung, 2016).

The TC2 purification algorithm is simple, robust, and rapidly convergent for closed-shell systems, and more efficient in orthogonal basis sets (Xiang et al., 2005). In this work, $H$ denotes the Hamiltonian matrix under the presentation of orthogonal basis sets. Reasonably in the preparatory step, a transformation $H=Z^{\mathrm{T}} H_{\mathrm{AO}} Z$ is required, here the matrix $Z$ is obtained by solving out the inverse square root of overlap matrix $S$ by the Cholesky factorization (Cholesky, 2005). The idempotency and
Algorithm 1 The pseudocode of TC2 algorithm, where $N_{e}$ is the number of electrons, $E$ is the energy-density matrix, $\varepsilon_{\min }(H)$ and $\varepsilon_{\max }(H)$ denote the minimal and maximum eigenvalue of the Hamiltonian matrix $H$, respectively.

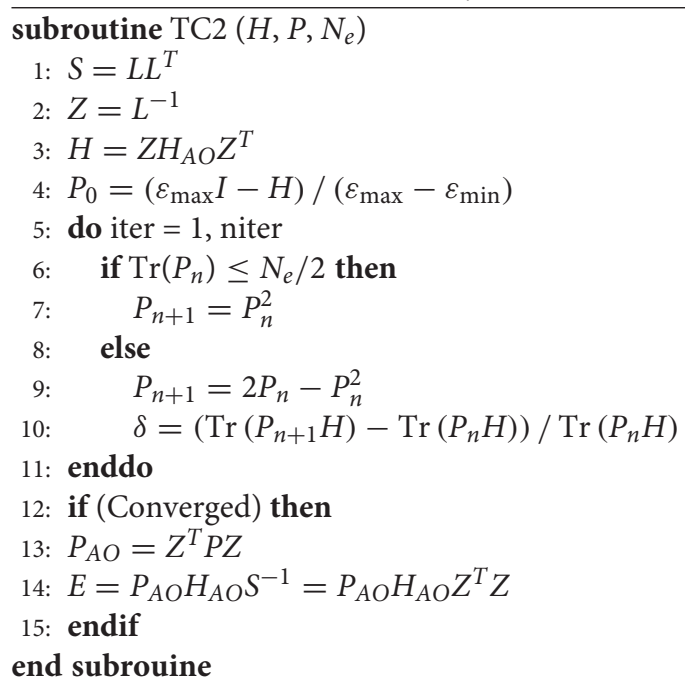

commutativity are satisfied naturally since the initial guess $P_{0}$ is obtained by the Lanczos method (Lanczos, 1950).

During each iteration step, the trace of $P_{n+1}$ is corrected by $P_{n}^{2}\left(\operatorname{Tr}\left(P_{n}\right) \leq N_{e} / 2\right)$ or $2 P_{n}-P_{n}^{2}\left(\operatorname{Tr}\left(P_{n}\right)>N_{e} / 2\right)$. Then, matrix elements less than a numerical threshold $\delta_{\text {filter }}\left(10^{-4}\right.$ or $\left.10^{-6}\right)$ are dropped to zero, thus maintaining the sparsity of density matrix. The pseudocode of the TC2 algorithm is given in Algorithm 1 and its corresponding owchart is shown in Figure $\mathbf{1 .}$

The density matrix purification method in HONPAS is based on the fact that both the density matrix and Hamiltonian matrix are sparse with NAOs. Therefore, sparse matrix multiplication is the most expensive step in the density matrix purification method. Figure 2 shows the sparsity of the density matrix $P$ saved as CSR format for the BN nanotubes consisting of 100 and 1,000 atoms (BNNT100 and BNNT1000) with different basis sets [single- $\zeta$ (SZ), double- $\zeta(\mathrm{DZ})$, and double- $\zeta$ plus polarization (DZP) $]$ and thresholds $\left(\delta_{\text {filter }}=10^{-4}\right.$ and $\left.10^{-6}\right)$ of non-zero elements in the density matrix $P$.

When the selected basis sets are strictly localized, the coefficient matrix $C$ for BNNT100 and BNNT1000 systems formed in a similar block diagonal matrix and arbitrary row of the resultant $P$ is occupied by same number of non-zero elements since $P=C C^{T}$. Therefore, the total number of nonzero elements grows linearly with the system size under tight binding approximation, which is the substantial precondition of almost all linear scaling algorithms. If we have an observation onto the first two columns of Figure 2, matrices show blockmultidiagonal patterns and sparse degree of BNNT 1000 with the SZ basis set under $\delta_{\text {filter }}=10^{-4}$ is $11.6 \%$, which is obviously less than that of BNNT100 (87.7\%). Variation trend of sparse degree is consistent with the character mentioned above from a perspective of image. Moreover, the cutoff radius $r_{c}$ of the DZP basis set is higher, so non-zero elements per row distribute more intensively 

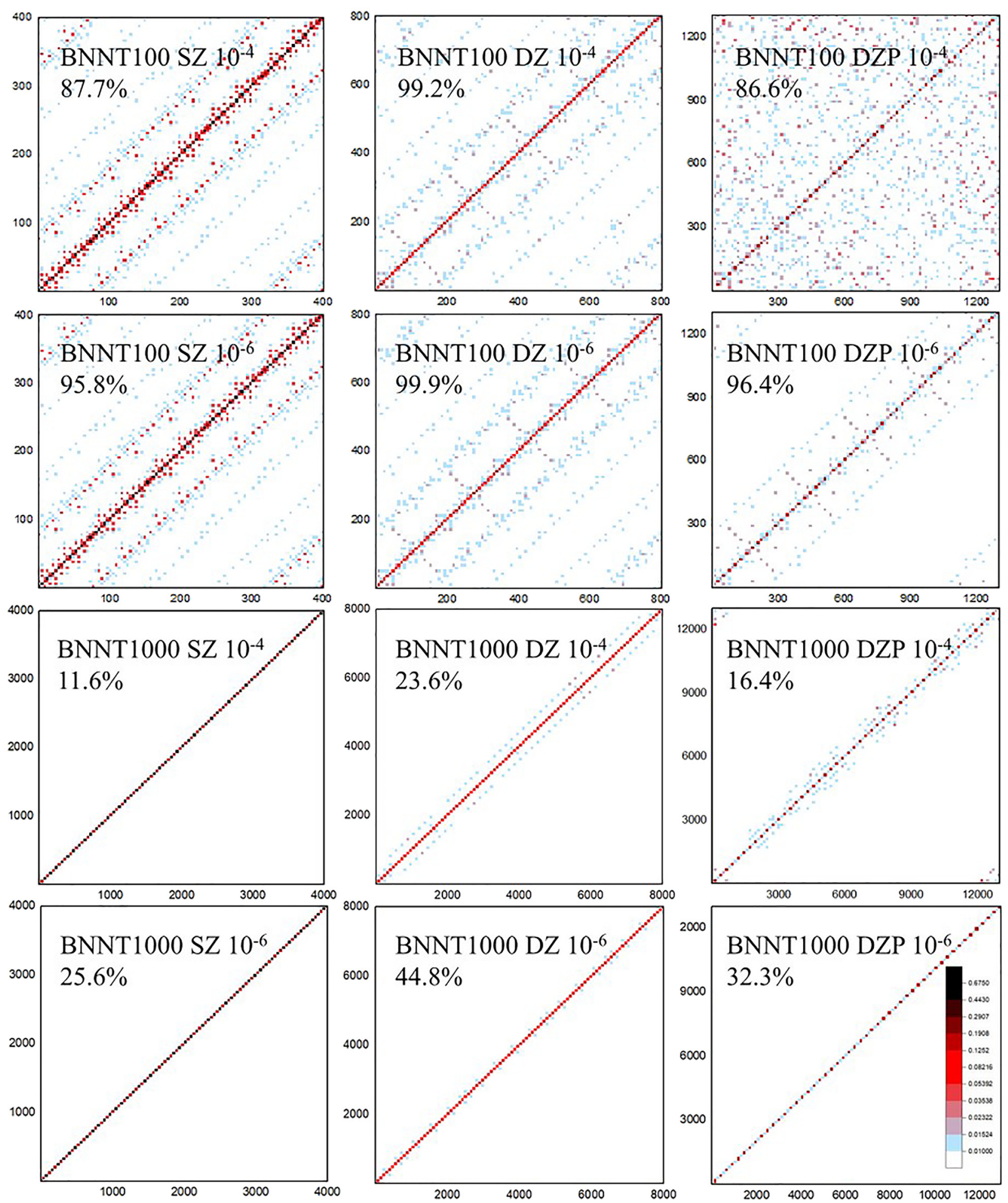

FIGURE 2 | The sparsity of the density matrix $P$ in the compressed sparse row (CSR) format of BN nanotubes (BNNTs) consisting of 100 and 1,000 atoms (BNNT100 and BNNT1000). Percentage under the system name indicates sparse degree of $P$. The matrix elements exceeding $10^{-2}$ are labeled as light blue and those exceeding $10^{-1}$ are marked as red. White area is remained for elements with tiny values or zero. Density matrix becomes less occupied with its elements gathering close to diagonal when the threshold after multiplication $100 \times$ tighter $\left(10^{-6}\right)$ than that of higher occupied matrix $\left(10^{-4}\right)$. When the basis sets become larger from SZ to DZP, sparse degree varies and $\delta_{\text {filter }}$ strongly affects the sparsity pattern. 
but their value is smaller than that of SZ and DZ. Sparse degree of BNNT1000 also decreases significantly from that of BNNT100 in the case of DZP basis set, such as $86.6 \%$ of BNNT100 and $16.4 \%$ of BNNT1000 at the same threshold, respectively.

On the perspective of threshold as shown in Figure 2, we observe that, as the system size increases, the influence of $\delta_{\text {filter }}$ is more significant, either the patterns or the sparse degree display an obvious difference under $\delta_{\text {filter }}=10^{-4}$ and $10^{-6}$. For example, the density matrix of BNNT1000 with the DZP basis set is $32.3 \%$ occupied under $\delta_{\text {filter }}=10^{-6}$ and $16.4 \%$ occupied under $\delta_{\text {filter }}$ $=10^{-4}$. On the other hand, $\delta_{\text {filter }}$ has a slight influence on the sparsity of BNNT100 compared with BNNT1000, which implies that the distribution of numerical value of matrix elements is shifted to higher level than that of BNNT1000. Just as elements dotted with deep color in BNNT100 are much more intensive than those of BNNT1000. It should be noted that dropping matrix elements less than $\delta_{\text {filter }}$ and using strictly truncated NAOs both contribute to the sparsity of $P$.

\subsection{Parallel Implementation of Sparse Matrix Multiplication}

The time required to process matrix-matrix multiplications during each iteration step accounts for a major part of total time. Note that there are some additional steps such as data communication and matrix addition. Fortunately, all matrices we need to deal with are sparse, so that the number of dot products reduces. The linear scaling cost arises from the fact that all matrix operations are performed on sparse matrices, which has a number of non-zero entries that increase linearly with the system size (VandeVondele et al., 2012).

The sparsity of matrix also causes unexpected drawbacks. An apparent disadvantage is, the matrix multiplication step would change the sparsity pattern during the self-consistent field (SCF) iterations, resulting in a load imbalance between matrix computation and data commutation among different processing cores. Since each matrix is distributed on a series of processes in advance, the instability of sparsity pattern will occur at each iteration, thus we also need to modify the data distribution after each iteration or exploit a block-cyclic distribution scheme. Apart from those, dropping matrix elements with the numerical value less than a threshold can reduce the number of dot products. But the computational accuracy of total energy and atomic forces is sacrificed inevitably under a loose threshold. The parallel version of TC2 algorithm in HONPAS is based on CSR data format and message-passing interface (MPI), which is capable of performing massive parallelism on modern heterogeneous supercomputers. We employ the SPARSEKIT library to manipulate and deal with sparse matrices, which provides programs for converting data structures, filtering out elements, and performing basic linear algebra operations with sparse matrix (Saad, 1994).

In the parallel TC2 module, there is a single hierarchical structure of parallelization that consists of single type of data distribution and communication scheme. The TC2 module utilizes the MPI parallel programming to deal with data communications between different MPI processes. In our work, the MPI processes are organized in $1 \mathrm{D}$ row MPI grids. The

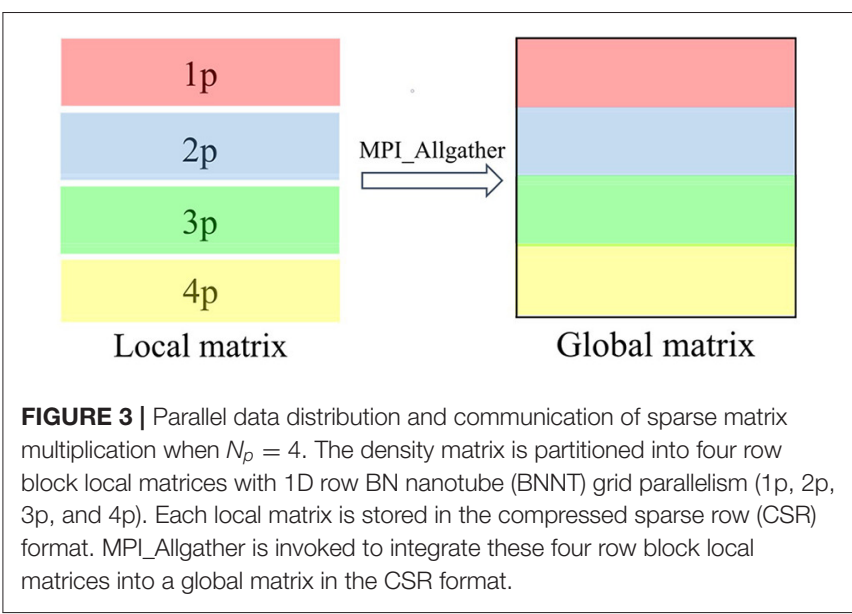

density matrix is distributed by $1 \mathrm{D}$ row blocks across MPI processes, and each process saves $N_{\mathrm{b}} / N_{\mathrm{p}}$ rows of global matrix. Thus, such local and global sparse matrix-matrix multiplication does not require additional data communication. Individual process computes its part of the multiplication, processing a row block of $\mathrm{np}\left(n=1,2, \ldots, N_{\mathrm{p}}\right)$ at a time. After the local multiplication has been processed, each processor just gathers a local subset of global density matrix. We use the MPI_Allgather function to gather local matrices into global density matrix in each MPI process, similar to the case of MPI_Gather and then MPI_Bcast, then performing local sparse matrix-matrix multiplication at the next iteration step. Figure 3 illustrates the schematic diagram of MPI communication on CSR data format, in which we set $N_{\mathrm{p}}=4$ to simplify the discussion.

\section{RESULTS AND DISCUSSION}

In this section, we demonstrate the computational accuracy and efficiency of our parallel TC2 algorithm. We implement this method in the HONPAS package (Qin et al., 2015), which has been written in the Fortran programming language with the MPI for parallelism. We use the norm-conserving Troullier-Martins pseudopotentials (Troullier and Martins, 1991) to represent interaction between core and valence electrons. We use the exchange-correlation functional of local density approximation of Goedecker-Teter-Hutter (LDA-PZ) (Goedecker et al., 1996) to describe the electronic structures of these BNNTs with a grid cutoff of 100 Ry. In our calculations, the NAOs are generated by default parameters in SIESTA. We utilize the linear-scaling density matrix TC2 purification algorithm to calculate the electronic structures of a series of boron nitride nanotubes (BNNTs), containing 100-18,000 atoms (labeled by BNNT100-BNNT18000). The details of the input parameters and atomic structures of BNNTs used in this work as well as the performance data are shown in the Supplementary Materials. 
TABLE 1 | Absolute error of total energy $\Delta E_{\text {tot }}$ (eV/atom) and the maximum of root mean square error of atomic forces $\Delta F_{\max }(\mathrm{eV} / \AA)$ of the TC2 method with varying thresholds of $\delta_{\text {filter }}=10^{-4}$ and $10^{-6}$ for the BNNT100 system with SZ, DZ, and DZP basis sets.

\begin{tabular}{lccc}
\hline Basis sets & $\delta_{\text {filter }}$ & $\boldsymbol{\Delta} \boldsymbol{E}_{\text {tot }}$ & $\boldsymbol{\Delta} \boldsymbol{F}_{\text {max }}$ \\
\hline $\mathrm{SZ}$ & $10^{-4}$ & $3.36 \times 10^{-4}$ & $8.48 \times 10^{-3}$ \\
DZ & $10^{-4}$ & $2.26 \times 10^{-3}$ & $9.80 \times 10^{-2}$ \\
DZP & $10^{-4}$ & $8.80 \times 10^{-3}$ & $3.07 \times 10^{-1}$ \\
SZ & $10^{-6}$ & $3.70 \times 10^{-7}$ & $3.64 \times 10^{-5}$ \\
DZ & $10^{-6}$ & $4.72 \times 10^{-5}$ & $1.73 \times 10^{-4}$ \\
DZP & $10^{-6}$ & $1.06 \times 10^{-5}$ & $4.30 \times 10^{-4}$
\end{tabular}

The reference results are computed by the direct diagonalization method.

\subsection{Accuracy}

We benchmark the computational accuracy of parallel TC2 method by comparing the results with those obtained from the diagnonalization method. We consider the effects of both the size of basis sets (SZ, DZ, and DZP) and different values of thresholds $\left(\delta_{\text {filter }}=10^{-4}\right.$ to $\left.10^{-6}\right)$ on the computational accuracy of density matrix TC2 purification algorithm. We define the errors of total energy and atomic forces, respectively, as

$$
\begin{aligned}
\Delta E_{\text {tot }} & =\left|\Delta E^{\mathrm{TC} 2}-E^{\mathrm{DIAG}}\right| / N_{\mathrm{A}} \\
\Delta F_{I} & =\left|F_{I}^{\mathrm{TC} 2}-F_{I}^{\mathrm{DIAG}}\right|
\end{aligned}
$$

where $N_{A}$ is the total number of atoms and $I$ is the atom index.

In the HONPAS calculations, the default convergence accuracy for total energy and atomic forces are $10^{-4} \mathrm{eV} /$ atom and $0.02 \mathrm{eV} / \AA$, respectively. Table 1 shows that the TC2 purification calculation for total energy is performed very well when choosing a tight dropping threshold, and $\delta_{\text {filter }}=10^{-6}$ can yield a total energy accuracy of $10^{-5} \mathrm{eV} /$ atom at least. On the other hand, strictly truncated NAOs can yield the sparsity without loss of accuracy simultaneously (Shang et al., 2010). We compute the total energy and atomic forces under different basis sets using a variable threshold. As shown in Figure 4, the errors of atomic forces from TC2 and those obtained from direct diagonalization method are indistinguishable. For all tested systems, the accuracy of the TC2 method can be obviously improved by tightening the threshold $\left(10^{-4}\right.$ to $\left.10^{-6}\right)$. In particular, when the threshold is set to $10^{-4}, \Delta F_{\max }$ with the most general case of DZP basis set reaches $10^{-1} \mathrm{eV} / \AA$, which is already comparable to the magnitude of atomic force itself. In contrast, $\Delta F_{\max }=4.30 \times$ $10^{-4} \mathrm{eV} / \AA$ under $\delta_{\text {filter }}=10^{-6}$ with the same basis set. The noticeable error arises from the lack of information in density matrix when too many elements are neglected after each iteration step and the information of Hamiltonian matrix just included in the initial step. However, the relative error of energy per atom is less than $10^{-7}$ when threshold is set to $10^{-6}$ in the case of SZ basis set, which indicates that the computational accuracy of TC2 method is still guaranteed. On the perspective of basis sets, high accuracy is ensured when we employ rigorously localized basis sets (SZ). Note that systems with the DZP basis set have relatively larger errors, since the information of polarization orbital is

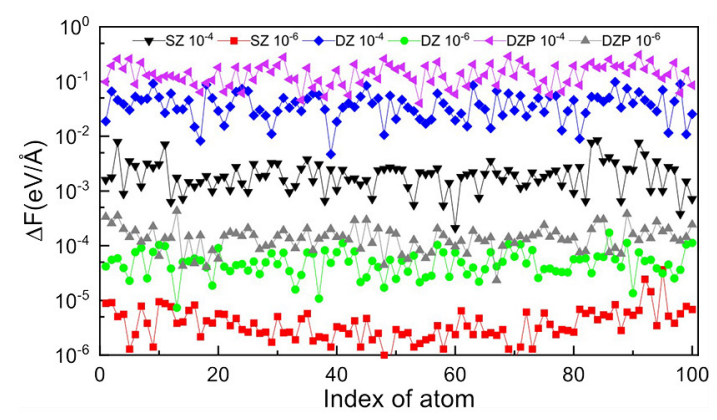

FIGURE 4 | Variation of root mean square error of atomic force on each atom computed with the TC2 and diagonalization methods with different basis sets (SZ, DZ, and DZP) and thresholds $\left(\delta_{\text {filter }}=10^{-4}\right.$ and $\left.10^{-6}\right)$ for BNNT100.

partly omitted by dropping matrix elements. For instance, when $\delta_{\text {filter }}$ is set to $10^{-4}$, the energy error for SZ is $3.36 \times 10^{-4}$ but that for DZP is $8.80 \times 10^{-3}$ (still can achieve the converged accuracy). As we have mentioned in section 2, non-zero elements those hold relatively small value distribute more intensively in the case of DZP basis set, and the physical information can be seriously lacking under relatively large $\delta_{\text {filter }}$.

\subsection{Efficiency}

We demonstrate the computational efficiency and parallel scalability of linear-scaling TC2 method by checking the weak and strong scaling performance on BNNT systems with the SZ basis set and a threshold of $\delta_{\text {filter }}=10^{-4}$. We illustrate the total time of the main time-consuming parts as shown in Figure 1: (a) Construction of Hamiltonian matrix, (b) evaluation of density matrix $P$ from Hamiltonian matrix by Cholesky factorization following Lanczos method, and (c) purification with matrix multiplication and addition. It should be noticed that the data communication via MPI interface also occupies numerous time resource while performing massive parallelization over plenty of processing cores. Practical tests on the computational efficiency and parallel scalability are performed in the case of BNNT systems with MPI parallelism on modern heterogeneous supercomputers, including comparison of TC2 and diagonalization methods with respect to different system sizes and process counts, as shown in Figures 5, 6, respectively. There are some additional steps such as computing total energy and atomic forces, which are all included in the total wall clock time of outer SCF iterations in the TC2 method.

Since the computational cost of linear-scaling TC2 method grows linearly with respect to the system size, a noteworthy speed up is supposed to be observed. We choose all tested systems with the SZ basis set to illustrate strong and weak scaling behaviors, since it is more strictly localized, resulting in a relatively small change of sparsity pattern after each iteration step. The variation of total time with respect to the system size is plotted in Figure 5. We can see that the scaling of TC2 is fitted to $O(N)$ due to the linearly growing sparse degree of $P$, and the number required to perform multiplication has the same trend. Linear scaling behavior is obtained with various systems containing 2,000-9,000 


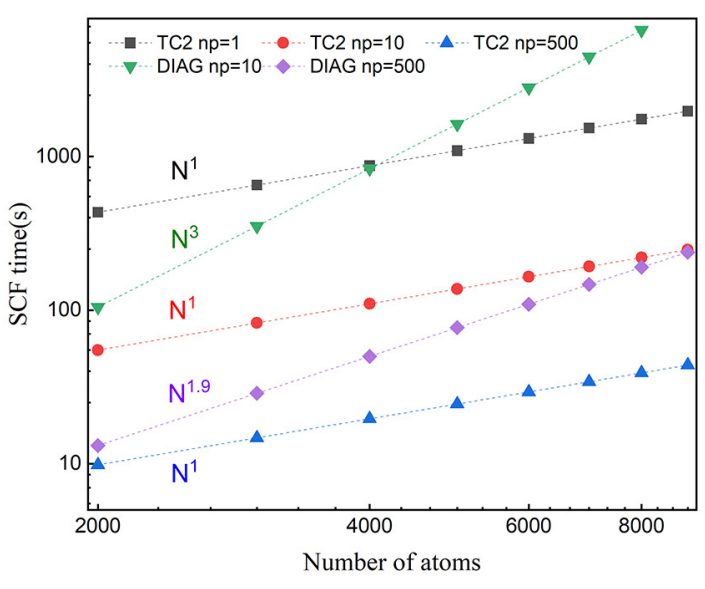

FIGURE 5 | Weak scaling of wall clock time per SCF iteration with respect to the number of atoms with the message-passing interface (MPI) parallelism for BN nanotubes (BNNTs) with 2,000 and 9,000 atoms (BNNT2000 and BNNT9000) computed with the TC2 and diagonalization methods.

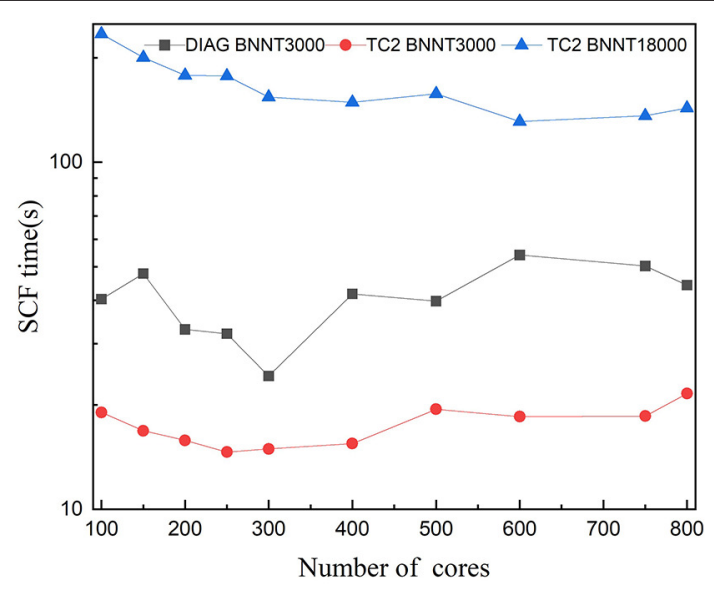

FIGURE 6 | Strong scaling of wall clock time per SCF iteration with respect to the number of cores with the message-passing interface (MPI) parallelism for BN nanotubes (BNNTs) with 3,000 and 18,000 atoms (BNNT3000 and BNNT18000) computed with the TC2 and diagonalization methods.

atoms under serial mode $\left(N_{p}=1\right)$, and it continues to scale further up to 500 cores at least, which benefits from the efficient parallel implementation of matrix multiplications based on the CSR formatted sparse density matrix. A speed-up of 4.7 can be achieved for 9,000 atoms (500 cores) and could be larger for more atoms. The fitted scaling for explicit diagonalization is just $O\left(N^{1.9}\right)$ with number of atoms fewer than 5,000 when the number of processors is relatively large, arising from the load imbalance that problem size (number of computational tasks) distributed on each process is not adequate and some cores remain idle. If the processors keep increasing, low efficiency of parallelization is going to happen. When the size of system grows sufficiently or processing cores have a relevant scale, fitted scaling turns back to $O\left(N^{3}\right)$ due to the cubic scaling of conventional diagonalization step. As a conclusion, linear-scaling TC2 method outperforms explicit diagonalization in terms of expansibility to large systems and massive parallel implementation.

Figure 6 compares the parallel scalability of TC2 to diagonalization methods. As it can be seen, the parallel scalability of both methods is unsatisfactory, especially with the smaller system size. This issue arises in the load imbalance caused by idle processors since computational tasks are inadequate compared with hundreds of cores. Test for 18,000 atoms with diagonalization is not represented due to a memory overflow problem (the dimension of matrix is 72,000). Unlike the diagonalization method, test for TC2 has been performed since the utilization of CSR data format reduces the memory requirement. TC2 method demonstrates just scaling up to 600 cores, since the $1 \mathrm{D}$ processes layout prevents it from massive parallelization. The performance of global MPI communications such as MPI_Allgather is strongly impacted by the physical distance of remote processing cores, which prompts us to utilize BCSR storage format and 2D block-cyclic processor layout.

\section{CONCLUSION AND OUTLOOK}

In summary, we present a parallel implementation of linearscaling density matrix trace correcting (TC) purification algorithm to solve KS equations with numerical atomic orbitals in the HONPAS package. We use the MPI_Allgather function for parallel programming to deal with the sparse matrix multiplication within the CSR format, which can scale up to hundreds of processing cores on modern heterogeneous supercomputers. We demonstrate the computational accuracy and efficiency of this linear-scaling density matrix purification algorithm by performing large-scale DFT calculations on boron nitrogen nanotubes containing tens of thousands of atoms.

However, our parallel implementation of TC2 method in HONPAS is inferior to that of BigDFT (Genovese et al., 2008; Mohr et al., 2014), ONETEP (Skylaris et al., 2005), and CONQUEST (Gillan et al., 2007). They exploit more than one level of organization and data distribution schemes resembling the BCSR format to handle the groups of atoms, which achieve high flexibility in load balancing (Bowler et al., 2002) with high performance on modern heterogeneous supercomputers. In the future, We plan to implement a massively parallel algorithm based on the NTPoly library (Dawson and Nakajima, 2018) in HONPAS. The NTPoly library utilizes the 3D sparse matrix multiplication algorithm, that is, the processors are organized into a three dimensional, cube-shaped virtual topology. In this case, density matrix purification algorithms can scale up to thousands of processing cores on modern heterogeneous supercomputers.

\section{DATA AVAILABILITY STATEMENT}

All datasets presented in this study are included in the article/Supplementary Material. 


\section{AUTHOR CONTRIBUTIONS}

ZL has tested the computational accuracy and efficiency of the TC2 method in HONPAS package, written manuscript and drawn figures. WH and XQ provide the research direction and revise the manuscript. $\mathrm{WH}$ has provided codes of the parallel version of TC2 method in HONPAS and directive methods for tests of computational accuracy and efficiency. LW has offered help for the modification of formulas. All authors contributed to the article and approved the submitted version.

\section{FUNDING}

This work was partly supported by the National Natural Science Foundation of China (21688102, 21803066), the Chinese Academy of Sciences Pioneer Hundred Talents Program (KJ2340000031), the National Key Research and Development Program of China (2016YFA0200604), the Anhui Initiative

\section{REFERENCES}

Banerjee, A. S., Lin, L., Hu, W., Yang, C., and Pask, J. E. (2016). Chebyshev polynomial filtered subspace iteration in the discontinuous Galerkin method for large-scale electronic structure calculations. J. Chem. Phys. 145:154101. doi: $10.1063 / 1.4964861$

Blum, V., Gehrke, R., Hanke, F., Havu, P., Havu, V., Ren, X., et al. (2009). Ab initio molecular simulations with numeric atom-centered orbitals. Comput. Phys. Commun. 180, 2175-2196. doi: 10.1016/j.cpc.2009.06.022

Borštnik, U., VandeVondele, J., Weber, V., and Hutter, J. (2014). Sparse matrix multiplication: The distributed block-compressed sparse row library. Parallel Comput. 40, 47-58. doi: 10.1016/j.parco.2014.03.012

Bowler, D., Miyazaki, T., and Gillan, M. (2002). Recent progress in linear scaling ab initio electronic structure techniques. J. Phys. Condens. Matter 14:2781. doi: $10.1088 / 0953-8984 / 14 / 11 / 303$

Bowler, D. R., and Miyazaki, T. (2012). O(N) methods in electronic structure calculations. Rep. Prog. Phys. 75:036503. doi: 10.1088/0034-4885/75/3/036503

Cankurtaran, B. O., Gale, J., and Ford, M. (2008). First principles calculations using density matrix divide-and-conquer within the SIESTA methodology. J. Phys. Condens. Matter 20:294208. doi: 10.1088/0953-8984/20/29/294208

Challacombe, M. (2014). Linear scaling solution of the timedependent self-consistent-field equations. Computation 2, 1-11. doi: $10.3390 /$ computation 2010001

Chen, M., Guo, G., and He, L. (2010). Systematically improvable optimized atomic basis sets for ab initio calculations. J. Phys. Condens. Matter 22:445501. doi: 10.1088/0953-8984/22/44/445501

Chen, M., Guo, G., and He, L. (2011). Electronic structure interpolation via atomic orbitals. J. Phys. Condens. Matter 23:325501. doi: 10.1088/0953-8984/23/32/325501

Cholesky, A.-L. (2005). Sur la résolution numérique des systémes d'équations linéaires. Bulletin de la Sabix. Société des amis de la Bibliothèque et de l'Histoire de l'École polytechnique 39, 81-95. doi: 10.4000/sabix.529

Corsetti, F. (2014). The orbital minimization method for electronic structure calculations with finite-range atomic basis sets. Comput. Phys. Commun. 185, 873-883. doi: 10.1016/j.cpc.2013.12.008

Daniels, A. D., and Scuseria, G. E. (1999). What is the best alternative to diagonalization of the Hamiltonian in large scale semiempirical calculations? J. Chem. Phys. 110, 1321-1328. doi: 10.1063/1.478008

Daw, M. S. (1993). Model for energetics of solids based on the density matrix. Phys. Rev. B 47:10895. doi: 10.1103/PhysRevB.47.10895

Dawson, W., and Nakajima, T. (2018). Massively parallel sparse matrix function calculations with NTPoly. Comput. Phys. Commun. 225, 154-165. doi: 10.1016/j.cpc.2017.12.010 in Quantum Information Technologies (AHY090400), the Strategic Priority Research Program of Chinese Academy of Sciences (XDC01040100), the Fundamental Research Funds for the Central Universities (WK2340000091), the Research Start-Up Grants (KY2340000094), and the Academic Leading Talents Training Program (KY2340000103) from University of Science and Technology of China. The authors thank the Supercomputing Center of Chinese Academy of Sciences, the Supercomputing Center of USTC, the National Supercomputing Center in Kunshan, Wuxi, Shanghai, and Guangzhou for the computational resources.

\section{SUPPLEMENTARY MATERIAL}

The Supplementary Material for this article can be found online at: https://www.frontiersin.org/articles/10.3389/fchem. 2020.589910/full\#supplementary-material
Etter, S. (2020). Incomplete selected inversion for linear-scaling electronic structure calculations. arXiv [Preprint]. arXiv:2001.06211. Available online at: https://arxiv.org/abs/2001.06211

Frisch, M. J., Pople, J. A., and Binkley, J. S. (1984). Self-consistent molecular orbital methods 25. Supplementary functions for Gaussian basis sets. J. Chem. Phys. 80, 3265-3269. doi: 10.1063/1.447079

Galli, G., and Parrinello, M. (1992). Large scale electronic structure calculations. Phys. Rev. Lett. 69:3547. doi: 10.1103/PhysRevLett.69.3547

Genovese, L., Neelov, A., Goedecker, S., Deutsch, T., Ghasemi, S. A., Willand, A., et al. (2008). Daubechies wavelets as a basis set for density functional pseudopotential calculations. J. Chem. Phys. 129:014109. doi: $10.1063 / 1.2949547$

Gillan, M. J., Bowler, D. R., Torralba, A. S., and Miyazaki, T. (2007). Order-N first-principles calculations with the CONQUEST code. Comput. Phys. Commun. 177, 14-18. doi: 10.1016/j.cpc.2007. 02.075

Goedecker, S. (1999). Linear scaling electronic structure methods. Rev. Mod. Phys. 71:1085. doi: 10.1103/RevModPhys.7 1.1085

Goedecker, S., and Colombo, L. (1994). Efficient linear scaling algorithm for tight-binding molecular dynamics. Phys. Rev. Lett. 73:122. doi: 10.1103/PhysRevLett.73.122

Goedecker, S., and Teter, M. (1995). Tight-binding electronic-structure calculations and tight-binding molecular dynamics with localized orbitals. Phys. Rev. B 51:9455. doi: 10.1103/PhysRevB.51.9455

Goedecker, S., Teter, M., and Hutter, J. (1996). Separable dual-space Gaussian pseudopotentials. Phys. Rev. B 54:1703. doi: 10.1103/PhysRevB. 54.1703

Ho, G. S., Lignères, V. L., and Carter, E. A. (2008). Introducing PROFESS: a new program for orbital-free density functional theory calculations. Comput. Phys. Commun. 179, 839-854. doi: 10.1016/j.cpc.2008.07.002

Hohenberg, P., and Kohn, W. (1964). Inhomogeneous electron gas. Phys. Rev. 136, B864-B871. doi: 10.1103/PhysRev.136.B864

Hu, W., Lin, L., and Yang, C. (2015a). DGDFT: a massively parallel method for large scale density functional theory calculations. J. Chem. Phys. 143:124110. doi: $10.1063 / 1.4931732$

Hu, W., Lin, L., and Yang, C. (2015b). Edge reconstruction in armchair phosphorene nanoribbons revealed by discontinuous Galerkin density functional theory. Phys. Chem. Chem. Phys. 17, 31397-31404. doi: $10.1039 /$ C5CP00333D

Kim, J., and Jung, Y. (2016). A perspective on the density matrix purification for linear scaling electronic structure calculations. Int. J. Quant. Chem. 116, 563-568. doi: 10.1002/qua.25048 
Kim, J., Mauri, F., and Galli, G. (1995). Total-energy global optimizations using nonorthogonal localized orbitals. Phys. Rev. B 52:1640. doi: 10.1103/PhysRevB.52.1640

Kohn, W., and Sham, L. J. (1965). Self-consistent equations including exchange and correlation effects. Phys. Rev. 140, A1133-A1138. doi: 10.1103/PhysRev.140.A1133

Kühne, T. D., Iannuzzi, M., Del Ben, M., Rybkin, V. V., Seewald, P., Stein, F., et al. (2020). CP2K: an electronic structure and molecular dynamics software package-Quickstep: efficient and accurate electronic structure calculations. J. Chem. Phys. 152:194103. doi: 10.1063/5.0007045

Lanczos, C. (1950). An Iteration Method for the Solution of the Eigenvalue Problem of Linear Differential and Integral Operators. United States Government Press Office, Los Angeles, CA. doi: 10.6028/jres.045.026

Li, X.-P., Nunes, R. W., and Vanderbilt, D. (1993). Density-matrix electronicstructure method with linear system-size scaling. Phys. Rev. B 47:10891. doi: 10.1103/PhysRevB.47.10891

Liang, W., Saravanan, C., Shao, Y., Baer, R., Bell, A. T., and Head-Gordon, M. (2003). Improved Fermi operator expansion methods for fast electronic structure calculations. J. Chem. Phys. 119, 4117-4125. doi: 10.1063/1.1590632

Lin, L., Lu, J., Ying, L., and Weinan, E. (2012). Adaptive local basis set for KohnSham density functional theory in a discontinuous Galerkin framework I: total energy calculation. J. Com. Phys. 231, 2140-2154. doi: 10.1016/j.jcp.2011.11.032

Mauri, F., and Galli, G. (1994). Electronic-structure calculations and moleculardynamics simulations with linear system-size scaling. Phys. Rev. B 50:4316. doi: 10.1103/PhysRevB.50.4316

Mohr, S., Ratcliff, L. E., Boulanger, P., Genovese, L., Caliste, D., Deutsch, T., et al. (2014). Daubechies wavelets for linear scaling density functional theory. J. Chem. Phys. 140:204110. doi: 10.1063/1.4871876

Mulliken, R. S. (1955). Electronic population analysis on LCAO-MO molecular wave functions. I. J. Chem. Phys. 23, 1833-1840. doi: 10.1063/1.1740588

Niklasson, A. M. (2002). Expansion algorithm for the density matrix. Phys. Rev. B 66:155115. doi: 10.1103/PhysRevB.66.155115

Niklasson, A. M., Tymczak, C., and Challacombe, M. (2003). Trace resetting density matrix purification in $\mathrm{O}(\mathrm{N})$ self-consistent-field theory. J. Chem. Phys. 118, 8611-8620. doi: 10.1063/1.1559913

Nunes, R., and Vanderbilt, D. (1994). Generalization of the densitymatrix method to a nonorthogonal basis. Phys. Rev. B 50:17611. doi: 10.1103/PhysRevB.50.17611

Ordejón, P., Drabold, D. A., Martin, R. M., and Grumbach, M. P. (1995). Linear system-size scaling methods for electronic-structure calculations. Phys. Rev. B 51:1456. doi: 10.1103/PhysRevB.51.1456

Ozaki, T., and Kino, H. (2005). Efficient projector expansion for the $a b$ initio LCAO method. Phys. Rev. B 72:045121. doi: 10.1103/PhysRevB.72.0 45121

Palser, A. H., and Manolopoulos, D. E. (1998). Canonical purification of the density matrix in electronic-structure theory. Phys. Rev. B 58:12704. doi: 10.1103/PhysRevB.58.12704

Pratapa, P. P., Suryanarayana, P., and Pask, J. E. (2016). Spectral quadrature method for accurate $\mathrm{O}(\mathrm{N})$ electronic structure calculations of metals and insulators. Comput. Phys. Commun. 200, 96-107. doi: 10.1016/j.cpc.2015.11.005
Qin, X., Shang, H., Xiang, H., Li, Z., and Yang, J. (2015). HONPAS: a linear scaling open-source solution for large system simulations. Int. J. Quant. Chem. 115, 647-655. doi: 10.1002/qua. 24837

Saad, Y. (1994). PARSEKIT: A Basic Tool Kit for Sparse Matrix Computation (version2). Urbana, IL: University of Illinois.

Shang, H., Xiang, H., Li, Z., and Yang, J. (2010). Linear scaling electronic structure calculations with numerical atomic basis set. Int. Rev. Phys. Chem. 29, 665-691. doi: 10.1080/0144235X.2010.520454

Skylaris, C.-K., Haynes, P. D., Mostofi, A. A., and Payne, M. C. (2005). Introducing ONETEP: linear-scaling density functional simulations on parallel computers. J. Chem. Phys. 122:084119. doi: 10.1063/1.1839852

Soler, J. M., Artacho, E., Gale, J. D., García, A., Junquera, J., Ordejón, P., et al. (2002). The SIESTA method for ab initio order-N materials simulation. J. Phys. Condens. Matter 14:2745. doi: 10.1088/0953-8984/14/11/302

Suryanarayana, P. (2013). On spectral quadrature for linear-scaling density functional theory. Chem. Phys. Lett. 584, 182-187. doi: 10.1016/j.cplett.2013.08.035

Suryanarayana, P. (2017). On nearsightedness in metallic systems for O(N) density functional theory calculations: a case study on aluminum. Chem. Phys. Lett. 679, 146-151. doi: 10.1016/j.cplett.2017.04.095

Troullier, N., and Martins, J. L. (1991). Efficient pseudopotentials for plane-wave calculations. Phys. Rev. B 43:1993. doi: 10.1103/PhysRevB.43.1993

VandeVondele, J., Borstnik, U., and Hutter, J. (2012). Linear scaling self-consistent field calculations with millions of atoms in the condensed phase. J. Chem. Theory Comput. 8, 3565-3573. doi: 10.1021/ct200897x

Xiang, H., Liang, W., Yang, J., Hou, J., and Zhu, Q. (2005). Spin-unrestricted linear-scaling electronic structure theory and its application to magnetic carbon-doped boron nitride nanotubes. J. Chem. Phys. 123:124105. doi: $10.1063 / 1.2034448$

Yang, W. (1991). Direct calculation of electron density in density-functional theory. Phys. Rev. Lett. 66:1438. doi: 10.1103/PhysRevLett.66.1438

Yang, W., and Lee, T.-S. (1995). A density-matrix divide-and-conquer approach for electronic structure calculations of large molecules. J. Chem. Phys. 103, 5674-5678. doi: 10.1063/1.470549

Zhang, G., Lin, L., Hu, W., Yang, C., and Pask, J. E. (2017). Adaptive local basis set for Kohn-Sham density functional theory in a discontinuous Galerkin framework II: force, vibration, and molecular dynamics calculations. J. Com. Phys. 335, 426-443. doi: 10.1016/j.jcp.2016.12.052

Conflict of Interest: The authors declare that the research was conducted in the absence of any commercial or financial relationships that could be construed as a potential conflict of interest.

Copyright (c) 2020 Luo, Qin, Wan, Hu and Yang. This is an open-access article distributed under the terms of the Creative Commons Attribution License (CC BY). The use, distribution or reproduction in other forums is permitted, provided the original author(s) and the copyright owner(s) are credited and that the original publication in this journal is cited, in accordance with accepted academic practice. No use, distribution or reproduction is permitted which does not comply with these terms. 